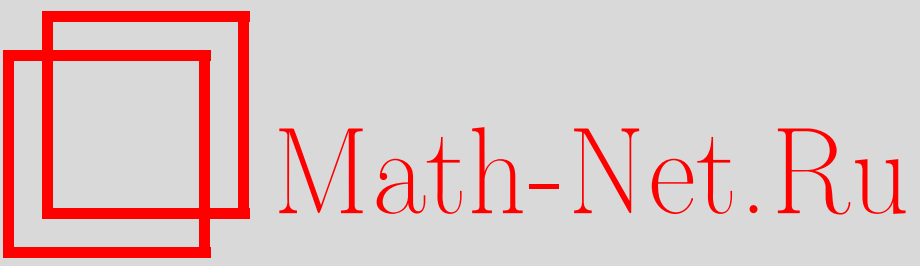

М.-М. Деза, М. И. Штогрин, Изометрические вложения полуправильных многогранников, разбиений и им дуальных в гиперкубы и кубические решетки, УМH, 1996, том 51, выпуск 6, 199-200

DOI: https://doi.org/10.4213/rm1026

Использование Общероссийского математического портала Math-Net.Ru подразумевает, что вы прочитали и согласны с пользовательским соглашением

http: //www . mathnet.ru/rus/agreement

Параметры загрузки:

IP : 54.198 .187 .58

26 апреля 2023 г., 06:44:02 


\title{
ИЗОМЕТРИЧЕСКИЕ ВЛОЖЕНИЯ ПОЛУПРАВИЛЬНЫХ МНОГОГРАННИКОВ, РАЗБИЕНИЙ И ИМ ДУАЛЬНЫХ В ГИПЕРКУБЫ И КУБИЧЕСКИЕ РЕШЕТКИ
}

\author{
М. ДЕзА, М.И. ШТоГРИн
}

Дан граф̆ $G=(V, E)$. Метрику кратчайшего пути в графе $G$ обозначим через $d_{G}$. Скажем, что $G$ вложим со шкалой $\lambda$ в гиперкуб $H_{m}$ или кубическую решетку $Z_{m}$, и назовем его $l_{1}$-графом (и обозначим это $G \rightarrow \frac{1}{\lambda} H_{m}$ или $G \rightarrow \frac{1}{\lambda} Z_{m}$ ), если существует отображение $\varphi$ множества вершин $V$ в $H_{m}$ или $Z_{m}$, при котором

$$
\lambda d_{G}\left(v_{i}, v_{j}\right)=\left\|\varphi\left(v_{i}\right)-\varphi\left(v_{j}\right)\right\|_{l_{1}}=\sum_{1 \leqslant k \leqslant m}\left|\varphi_{k}\left(v_{i}\right)-\varphi_{k}\left(v_{j}\right)\right|, \quad v_{i}, v_{j} \in V .
$$

При этом мы выбираем наименьшее такое число $\lambda$.

Важное необходимое условие для $G \rightarrow \frac{1}{\lambda} H_{m}$ или $G \rightarrow \frac{1}{\lambda} Z_{m}$ состоит в том [5], что метрика $d_{G}$ удовлетворяет всем гиперметрическим неравенствам

$$
\sum_{1 \leqslant i<j \leqslant n} b_{i} b_{j} d_{G}\left(v_{i}, v_{j}\right) \leqslant 0, \quad\left(b_{1}, b_{2}, \ldots, b_{n}\right)=b \in Z_{n}, \quad \sum_{1 \leqslant k \leqslant n} b_{k}=1 .
$$

При $b=(1,1,1,-1,-1)$ неравенство называется 5-гональныц.м. Для односвязной поверхности, например, все триангуляции типа [4.2m.2n], исключая октаэдр [4.4.4], невложимы, так как они не 5-гональны. Для многих архимедовых и им дуальных разбиений двумерной поверхности произвольного рода нарушаются те же 5-гональные неравенства, что и на универсальной накрьвающей.

Известно [1], что метрика с рациональными значениями изометрически вкладьвается в $l_{1}^{M}$ (т.е. $R^{M}$ с метрикой $\|x-y\|_{l_{1}}$ ) тогда и только тогда, когда она вложима изометрически с некоторой шкалой $\lambda$ в некоторый гиперкуб $H_{m}$. Любой планарный $l_{1}$-граф имеет шкалу 1 или 2 [4]. Конечные $l_{1}$-графы охарактеризованы в [12] и [6] как изометрические подграбы прямого произведения полукубов и $\beta_{n}$. Полиномиальньй алгорифм нахождения $l_{1}$-графов дан в [9]. Для $l_{1}$-графа с $n \geqslant 4$ вершинами либо $\lambda=1$, либо $\lambda \leqslant n-2$ четно [12]. При $n-2=2^{k}, k \in N$, эта оценка неулучшаема. В [7] показано, что остов конечного $n$-зонотопа, т.е. аффинной проекции гиперкуба, есть изометрический подграф куба, проекцией которого он является, и даны примеры центрально-симметричных многогранников, изометрично вложимых в куб, но не зоноэдров. Любое разбиение плоскости на центрально-симметричные многоугольники зоноэдрально и вложимо как в $H_{\infty}$, так и в $Z_{m}, m \leqslant \infty$. Например, хорошо известное непериодическое разбиение плоскости на ромбы, предложенное Пенроузом, вкладывается в $Z_{5}$, в связи с чем Де Брюйн представляет это разбиение как проекцию.

Пусть граф $G$ вложен в гиперкуб. Тогда любой паре противоположных гиперграней соответствует разрез графа $G$ на компоненты $G_{1}, G_{2}$, вложенные в эти грани. Два вложения графа $G$ назовем әквивалентными, если им соответствует одна и та же совокупность разрезов. Граф $G$ называется $l_{1}$-жестким [8], если все его вложения в гиперкубы попарно эквивалентны. Если $\lambda=1$, то $l_{1}$-грабф является $l_{1}$-жестким [8]. Для $l_{1}$-жесткого граффа $\lambda=1$ или $\lambda=2$ [12]. Любой $l_{1}$-граф̆, не содержащий $K_{n}$, является $l_{1}$-жестким [4].

Вложения правильных многогранников $\left(3^{3}\right) \rightarrow \frac{1}{2} H_{3},\left(3^{3}\right) \rightarrow \frac{1}{2} H_{4},\left(3^{4}\right) \rightarrow \frac{1}{2} H_{4},\left(3^{5}\right) \rightarrow$ $\frac{1}{2} H_{6},\left(4^{3}\right) \rightarrow H_{3},\left(5^{3}\right) \rightarrow \frac{1}{2} H_{10}$ впервые получены в [11]. В [2] найдены вложения правильных разбиений евклидовой плоскости $\left(3^{6}\right) \rightarrow \frac{1}{2} Z_{3},\left(6^{3}\right) \rightarrow \frac{1}{2} Z_{3}$ и показано, что правильные разбиения $\{2 n, p\}$ и $\{\infty, p\}$ гиперболической плоскости вложимы в $H_{\infty}$. Оставшийся случай нечетных многоугольников решает суммируюшая

ТеОрема 1. Остов любого правильного разбиения $S^{2}, R^{2}$ и $\Lambda^{2}$ вложим со икалой 1 или 2 в гиперкуб, конечномерный для конечного графа.

Работа выполнена при финансовой поддержке Российского фонда фундаментальных исследований (грант № 96-01-00166) и DFG (Рурский университет Бохум, университет Билефельд). 
Теорема 2. Среди архимедовых и дуальных им (каталановых) многогранников вложимы только следующие: $\left[3.6^{2}\right] \rightarrow \frac{1}{2} H_{7},\left(4.6^{2}\right) \rightarrow H_{6},\left[3.8^{2}\right] \rightarrow \frac{1}{2} H_{12},[3.4 .3 .4] \rightarrow H_{4}$, $(4.6 .8) \rightarrow H_{9},\left(3.4^{3}\right) \rightarrow \frac{1}{2} H_{10},\left(3^{4} .4\right) \rightarrow \frac{1}{2} H_{9},\left[5.6^{2}\right] \rightarrow \frac{1}{2} H_{10},\left[3.10^{2}\right] \rightarrow \frac{1}{2} H_{26},[3.5 .3 .5] \rightarrow H_{6}$, $(4.6 .10) \rightarrow H_{15},(3.4 .5 .4) \rightarrow \frac{1}{2} H_{16},\left(3^{4} .5\right) \rightarrow \frac{1}{2} H_{15} ;\left(4^{2} .2 n+1\right) \rightarrow \frac{1}{2} H_{2 n+3},\left(4^{2} .2 n\right) \rightarrow H_{n+1}$, $\left[3.4^{2}\right] \rightarrow \frac{1}{2} H_{4},\left(3^{3} . n\right) \rightarrow \frac{1}{2} H_{n+1}$.

Теорема 3. Среди архимедовых и им дуальных (лавесовских) разбиений плоскости вложимы только следующие: [3.6.3.6] $\rightarrow Z_{3},(3.4 .6 .4) \rightarrow \frac{1}{2} Z_{3},\left(4.8^{2}\right) \rightarrow Z_{4},(4.6 .12) \rightarrow Z_{6}$, $\left[3.12^{2}\right] \rightarrow \frac{1}{2} Z_{\infty},\left(3^{4} .6\right) \rightarrow \frac{1}{2} Z_{6},\left(3^{3} \cdot 4^{2}\right) \rightarrow \frac{1}{2} Z_{3},\left(3^{2} .4 .3 .4\right) \rightarrow \frac{1}{2} Z_{4}$

Теорема 4. Среди 2-униформных разбиений плоскости и им дуальных (см. [10, рис. 2.2.1]) вложими только следующие: $\left(3^{6} ; 3^{4} .6\right)_{1} \rightarrow \frac{1}{2} Z_{6},\left(3^{6} ; 3^{3} .4^{2}\right)_{1} \rightarrow \frac{1}{2} Z_{3}$, $\left(3^{6} ; 3^{3} \cdot 4^{2}\right)_{2} \rightarrow \frac{1}{2} Z_{3},\left(3^{6} ; 3^{2} .4 .3 .4\right) \rightarrow \frac{1}{2} Z_{6},\left(3^{4} \cdot 6 ; 3^{2} \cdot 6^{2}\right) \rightarrow \frac{1}{2} Z_{5},\left(3^{3} .4^{2} ; 3^{2} \cdot 4.3 .4\right)_{1} \rightarrow \frac{1}{2} Z_{6}$, $\left(3^{3} .4^{2} ; 3^{2} .4 .3 .4\right)_{2} \rightarrow \frac{1}{2} Z_{4},\left(3^{3} .4^{2} ; 3.4 .6 .4\right) \rightarrow \frac{1}{2} Z_{3},\left(3^{3} .4^{2} ; 4^{4}\right)_{1} \rightarrow \frac{1}{2} Z_{3},\left(3^{3} .4^{2} ; 4^{4}\right)_{2} \rightarrow \frac{1}{2} Z_{3}$, $\left(3^{6} ; 3^{2} .6^{2}\right)^{*} \rightarrow Z_{\infty},\left(3.4^{2} .6 ; 3.6 .3 .6\right)_{2}^{*} \rightarrow Z_{3}$.

Среди примеров $k$-униформных разбиений плоскости $R^{2}$, данных в [10] на рис. 2.2 .2$, и им дуальных вкладьваются только первое из них: $\left(3^{3} .4^{2} ; 3^{2} .4 .3 .4 ; 4^{4}\right) \rightarrow \frac{1}{2} Z_{4}$; на рис. 2.2 .3 - вложимы только $(a) \rightarrow \frac{1}{2} Z_{6}$ и $(b)^{*} \rightarrow \frac{1}{2} Z_{\infty}$; на рис. 2.1.6 ни само разбиение, ни ему дуальное не вложимо. Заметим, что те $l_{1}$-графы, которые являются остовами $k$-униформных и дуальных им разбиений сферы $S^{2}$, евклидовой плоскости $R^{2}$ или плоскости Лобачевского $\Lambda^{2}$, вложимы, соответственно, в конечномерный куб, конечномерную решетку или бесконечномерную решетку. Отметим также, что для любого архимедова многогранника, кроме $\left(3.4^{2}\right)$, или разбиения $R^{2}$ и ему дуального остов ровно одного из них является $l_{1}$-графом. Скрученный ромбокубооктаэдр Ашкинузе-Миллера и ему дуальный не 5-гональны.

Теорема 5 . Bce $l_{1}$-графы, фигурирующие в теоремах $1,2,3$ и 4 , за исключением двух графов $\left(3^{3}\right)$ u $\left[3.4^{2}\right]$, являются $l_{1}$-эсесткими.

Среди правильных многогранников при $n \geqslant 4$ вложимы $\alpha_{n}, \beta_{n}, \gamma_{n}$ и не вложимы 4-мерные 120-гранник [7], 600- и 24-гранник [2].

ТЕОрема 6. При $n \geqslant 4$ вложимы только следующие полуправильные многогранники ( в обозначениях Кожсетера): $O_{21} \rightarrow \frac{1}{2} H_{5}, 1_{21} \rightarrow \frac{1}{2} H_{5} u$, бить может, $s\{3,4,3\} \rightarrow \frac{1}{2} H_{12}$.

Вопрос о вложимости для всех 92 случаев 3-многогранников с правильными гранями рассмотрен в [7]. Используя список из [3], этот вопрос для $n \geqslant 4$ решает

ТеОрема 7. Среди многогранников с правильными гранями при $n \geqslant 4$ вложимь только пирамида на основании $\beta_{n-1}$, бипирамида на основании $\alpha_{n-1}$ и $O_{21}$ с пирамидой над одной октаәдрической гранью.

\section{СПИСОК ЛИТЕРАТУРЫ}

[1] Assouad P., Deza M. // Ann. Discrete Math. 1980. V. 8. P. 197-210. [2] Assouad P. // The Geometric Vein, the Coxeter Festschrift. 1981. P. 141-147. [3] Blind G., Blind R. // Comment. Math. Helv. 1991. V. 66. P. 150-154. [4] Chepoi V., Deza M., Grishukhin V. // Preprint LIENS-96-11. [5] Тылкин М.Е. // ДАН. 1960. Т. 134. №5. С. 1037-1040. [6] Deza M., Grishukhin V. // Quart. J. Math. Oxford. 1993. V. 2. P. 399-433. [7] Deza M., Grishukhin V. // Preprint LIENS-96-1. [8] Deza M., Laurent M. // J. Algebraic Combin. 1994. V. 3. P. 153-175. [9] Deza M., Shpectorov S. // European. J. Combin. 1996. V. 17. P. 279-289. [10] Grunbaum B., Shephard G. C. Tilings and Patterns. New York: Freeman and Company, 1986. [11] Kelly J. B. // Lect. Notes in Math. 1975. V. 490. P. 17-31. [12] Shpectorov S. // European. J. Combin. 1993. V. 14. P. 117-130.

LIENS, Ecole Normale Superieure and CNRS, Paris; Математический институт им. В.А. Стеклова РАН
Принято редколлегией 02.09.1996 